\title{
Clinical characteristics and survival of patients with three major connective tissue diseases associated with pulmonary hypertension: A study from China
}

\author{
JIE PAN, LING LEI, CHENG ZHAO, JING WEN, FANG QIN and FEI DONG \\ Department of Rheumatology and Immunology, The First Affiliated Hospital of Guangxi Medical University, \\ Nanning, Guangxi Zhuang Autonomous Region 530000, P.R. China
}

Received May 4, 2020; Accepted March 17, 2021

DOI: $10.3892 / \mathrm{etm} .2021 .10357$

\begin{abstract}
The present cross-sectional study investigated the clinical characteristics and survival of patients with three types of connective tissue disease associated with pulmonary hypertension (CTD-PH) diagnosed early by echocardiography. A total of 218 patients with CTD-PH were included in the present study. Patients with the three major types of CTD, namely systemic lupus erythematosus (SLE), systemic sclerosis (SSc) and primary Sjögren's syndrome (pSS), were included. PH was diagnosed based on pulmonary arterial systolic pressure $>35 \mathrm{mmHg}$, as measured by Doppler echocardiography. Demographic data, clinical features, laboratory results and echocardiographic parameters were collected and analyzed. The Kaplan-Meier method was used to calculate survival rates. Multivariate analysis was used to identify independent factors affecting mortality. Compared with patients with CTD with pSS (6.5\%) or SLE (3.8\%), those with SSc had a higher prevalance of PH (12.9\%). Patients with SSc-PH had the highest rate of lung involvement $(81.2 \%)$ and $42.2 \%$ of patients were classified as World Health Organization-function class III/IV at the time of diagnosis with $\mathrm{PH}$. The overall
\end{abstract}

Correspondence to: Dr Ling Lei, Department of Rheumatology and Immunology, The First Affiliated Hospital of Guangxi Medical University, 6 Shuangyong Road, Nanning, Guangxi Zhuang Autonomous Region 530000, P.R. China

E-mail: leiling1972@aliyun.com

Abbreviations: Ab, antibody; ALP, alkaline phosphatase; MCTD, mixed connective tissue disease; ESSDAI, EULAR Sjögren's syndrome disease activity index; HRCT, high-resolution computed tomography; Ig, immunoglobulin; ILD, interstitial lung disease; mRSS, modified Rodan skin score; NT-proBNP, N-terminal pro-brain natriuretic peptide; $\mathrm{PAH}$, pulmonary arterial hypertension; $\mathrm{PH}$, pulmonary hypertension; PASP, pulmonary arterial systolic pressure; pSS, primary Sjögren's syndrome; SLE, systemic lupus erythematosus; SLEDAI, SLE activity index score; SSc, systemic sclerosis; WHO-FC, World Health Organization-function class

Key words: SLE, systemic sclerosis, pSS, connective tissue disease, $\mathrm{PH}$ survival rate among patients with CTD-PH at 1,3 and 5 years was $81.4,72.4$ and $56.9 \%$, respectively. Patients with SLE-PH appeared to have the most favorable prognosis and patients with SSc-PH had the poorest relative outcomes. Multivariate analysis revealed that age $\geq 50$ years was the only independent risk factor for mortality. In conclusion, among the patients with CTDs investigated, the prevalence of $\mathrm{PH}$ was highest among those with SSc. Patients with SSc-PH had the highest prevalence of pulmonary involvement, the lowest survival rate and the worst prognosis.

\section{Introduction}

Pulmonary arterial hypertension (PAH) is characterized by progressive increases in pulmonary vascular resistance and pulmonary arterial pressure, which ultimately lead to right heart failure and death $(1,2)$. Registry data have indicated that connective tissue disease (CTD) is the second leading cause of PAH. Idiopathic PAH accounts for $46 \%$ of all cases and CTD associated with PAH (CTD-PAH) accounts for $25 \%$ (3). The prevalence of CTD-PAH differs among different populations. For instance, several studies from the US and Europe have investigated systemic sclerosis ( $\mathrm{SSc}$ )-associated $\mathrm{PAH}$ (SSc-PAH), which accounts for $\sim 75 \%$ of all CTD-PAH cases, followed by systemic lupus erythematosus (SLE)-PAH (8-19\%), mixed CTD (MCTD)-PAH (8-9\%) and primary Sjögren's syndrome (pSS)-PAH (1\%) (3). However, cohort studies from Japan (4), South Korea (5) and China $(6,7)$ indicated that SLE-PAH was the most common type of CTD, followed by SSc, pSS and MCTD. These results suggested that the clinical characteristics and prognosis of Asian patients with CTD-PAH may differ from those of patients with CTD-PAH in Western countries.

PAH is one of the leading causes of morbidity and mortality among patients with CTD-PAH (7). The 1- and 3-year survival rates among patients with CTD-PAH are $\sim 80$ and $50 \%$, respectively. Prognosis is less favorable for patients with SSc-PAH compared with that for patients with SLE-PAH in Western and Asian countries (6,7). A retrospective review of patients with SLE from Peking Union Medical College Hospital (PUMCH; Beijing, China) over the last 30 years revealed that pulmonary hypertension $(\mathrm{PH})$ was the third most common cause of 
death, after lupus encephalopathy and renal involvement (8). $\mathrm{PH}$ may occur in isolation or in association with interstitial lung disease (ILD), the latter of which is probably caused by pulmonary interstitial lesions, which increase resistance in the pulmonary circulation (1). Increased resistance in the pulmonary circulation impairs oxygen exchange, causing long-term hypoxia, which promotes the pathogenesis of $\mathrm{PH}$ (1). Previous studies have indicated that mortality is higher in patients with CTD-PH and lung involvement, compared with that in patients with isolated PH $(1,6,9)$. In the modern treatment era, targeted therapies have improved the outcomes for patients with $\mathrm{PH}$, including exercise capacity, World Health Organization-function class (WHO-FC) and hemodynamic parameters $(10,11)$. However, less satisfactory outcomes were observed after treatment in Chinese patients $(1-3,10,11)$. Therefore, early screening for $\mathrm{PH}$ and expedient diagnosis are important to improve the prognosis of patients with CTD-PAH.

Doppler echocardiography is a non-invasive technology and has been used routinely as a tool to screen for and detect early-stage PAH. Pulmonary arterial systolic pressure (PASP), as measured by echocardiography, has been indicated to correlate with hemodynamic parameters, as measured by right heart catheterization (RHC) $(1,5,12-14)$. The echocardiographic method most commonly used to detect PH estimates right ventricular systolic pressure based on maximal tricuspid regurgitation velocity (TRV). Patients with TRV $>3.4 \mathrm{~m}^{2}$ (corresponding to a PASP $>50 \mathrm{mmHg}$ ) or with TRV between 2.9 and $3.4 \mathrm{~m}^{2}$ (corresponding to a PASP between 35 and $49 \mathrm{mmHg}$ ) in the presence of other signs are considered to have PH. Echocardiography based on the estimation of PASP has an estimated sensitivity of $0.79-1.00$ and specificity of $0.6-0.98$ for the detection of PH, as confirmed by RHC (1,3,5,9-14). pSS was recognized as a major CTD-associated PAH in China and this trend remained consistent in South Korea and Japan $(4,5)$. Though the pathology of SLE, SSc and pSS is characterized as vasculitis in certain patterns, the difference in survival among SSc-, SLE- and pSS-associated PAH remains unexplained. Previous studies indicated that right cardiac insufficiency was a significant predictor of mortality for patients with CTD-PAH (3-5), but there is currently no consensus regarding prognostic risk factors such as age, Raynaud's phenomenon, pericardial effusion and ILD. Therefore, in the present study, the clinical characteristics of patients with three major CTDs (SLE, SSc and pSS) associated with early diagnosed PH were investigated and the associated controversial progressive factors were explored. Patients in a southwestern region of China were evaluated with Doppler echocardiography to provide a comprehensive understanding of CTD-PH and to facilitate the early recognition, prevention and treatment of $\mathrm{PH}$ in CTD.

\section{Materials and methods}

\section{Patient selection}

Inclusion criteria. A total of 4,153 hospitalized patients with one of the three major CTDs (3,133 SLE, 497 SSc and 523 pSS) were included in calculations of the prevalence rate. A total of 218 patients with CTD-PH (120 patients with SLE-PH, 64 patients with SSc-PH and 34 patients with pSS-PH) encountered at the First Affiliated Hospital of Guangxi Medical University (Nanning, China) between October 2012 and January 2018 were retrospectively included in the present study. SLE was diagnosed according to the American College of Rheumatology (ACR) criteria, which were revised in 1997 and 2012 (15). SSc was diagnosed according to the American Rheumatism Association criteria established in 1980 and confirmed by the ACR/European League Against Rheumatism (EULAR) classification criteria developed in 2013 (16). The SSc subtype was defined as limited cutaneous disease (lcSSc) if skin thickening was confined to distal extremities (below the elbows and knees) and above the clavicles. SSc was defined as diffuse cutaneous disease $(\mathrm{dcSSc})$ if skin thickening involved the proximal extremities and the torso (16). pSS was diagnosed according to the revised criteria proposed by the American-European Consensus Group in 2002 (17). All patients were $>14$ years of age.

The patients with the three major types of CTD were assessed by Doppler echocardiography at the time of first visit to our hospital with the presence or absence of symptoms in order to screen for and detect early-stage CTD-PH at the initial evaluation. PASP $>35 \mathrm{mmHg}$ was defined as early diagnosed CTD-PH and such patients were enrolled in the present study. According to the PASP, the severity of each patient's PH was classified as mild $(36-50 \mathrm{mmHg})$, moderate $(51-70 \mathrm{mmHg})$ or severe $(\geq 71 \mathrm{mmHg})(3,9,12)$. Echocardiography was performed to evaluate the right-heart morphology in all patients. The measurements obtained included right atrial and ventricular enlargement, as well as main pulmonary artery widening (diameter $>26 \mathrm{~mm}$ ). Lung involvement may affect pre-capillary arterioles in PAH or post-capillary venules in pulmonary veno-occlusive disease (3). As no right cardiac catheterization and pulmonary function tests were performed, it was not possible to confirm cases of isolated PAH. Patients with SSc, pSS with ILD or SLE who had lung involvement (such as diffuse alveolar hemorrhage and acute lupus pneumonia) were included in the study.

Exclusion criteria. Other CTDs associated with PH, including rheumatoid arthritis, MCTDs, Takayasu arteritis, adult-onset Still's disease and undifferentiated CTD, were excluded from the present study, as they were rarely encountered at our center. Patients with overlapping syndrome were also excluded from the study.

PH caused by other conditions described in the 2015 European Society of Cardiology and the European Respiratory Society guidelines (1) for the Diagnosis and Treatment of PH were excluded from the study: Definite history of idiopathic PAH, obstructive sleep apnea, chronic obstructive pulmonary disease, primary valvulopathy, pulmonary disease with a mixed restrictive or obstructive pattern, portal hypertension, left heart disease, drug/toxin exposure, HIV infection or any other diseases known to be associated with PH (1).

Data collection. Demographic data, baseline clinical features, laboratory results and echocardiographic parameters were collected for analysis. The data collected at the time of $\mathrm{PH}$ diagnosis included age, sex, duration of CTDs, duration from the onset of PH-associated symptoms (exertional dyspnea, chest pain, chest tightness and cough) to the initial diagnosis of PH, WHO-FC, serological autoantibody profiles, N-terminal pro-brain natriuretic peptide (NT-proBNP) levels and other biochemical parameters. Specific clinical manifestations were 
recorded, including dyspnea, cough, expectoration, edema, chest tightness, chest pain, Raynaud's phenomenon, ischemic ulcer/pit and pericardial effusion. High-resolution computed tomography (HRCT) scans were performed routinely to evaluate ILD and the severity of pulmonary fibrosis was scored individually. In brief, each lung was divided into three zones: Upper (lung apex to aortic arch), middle (aortic arch to inferior pulmonary veins) and lower (inferior pulmonary veins to lung base). The extent of pulmonary abnormality in each of these six zones was scored using a scale from 0 to 4 ( 0 , absent; 1, 1-25; 2, 26-50; 3, 51-75; and 4, 76-100\%). Pulmonary fibrosis was characterized as pure ground-glass opacity, pulmonary fibrosis or honeycomb cysts (18). The HRCT images were scored according to the research of Goldin et al (18) by two radiologists (>20 years working experience) independently, when discrepancies occurred, a consensus was reached after discussion. For each patient with CTD, the disease activity was scored: The SLE activity index score (SLEDAI) for patients with SLE (12); the modified Rodan skin score (mRSS) for SSc (19); and the EULAR Sjögren syndrome disease activity index (ESSDAI) for pSS (20). Any history of clinical treatment for CTD-PH, such as the use of glucocorticoids, immunosuppressants or pulmonary vasodilators, was also recorded. The initial dose of glucocorticoid [ $\leq 0.5$ and $1-2 \mathrm{mg} / \mathrm{kg} /$ day (d), as well as $0.5-1 \mathrm{~kg} / \mathrm{d}]$ was determined based on the patient's condition and level of CTD disease activity. The immunosuppressants used alone or in combination among patients with CTD included cyclophosphamide pulse [one pulse of $500 \mathrm{mg} / \mathrm{m}^{2}$ per month (mo) for 6-8 mo], azathioprine $(100 \mathrm{mg} / \mathrm{d})$, cyclosporine $(3-5 \mathrm{mg} / \mathrm{kg} / \mathrm{d}$, bid), methotrexate (10-15 mg, weekly), leflunomide ( $20 \mathrm{mg} /$ day), thalidomide $(50-75 \mathrm{mg} /$ day), mycophenolate mofetil $(75 \mathrm{mg}$, bid) and tripterygium glycosides (50 mg, tid). The PAH-specific therapies administered included endothelial receptor antagonists (bosentan and ambrisentan), phosphodiesterase type 5 inhibitors (sildenafil, tadalafil), and beraprost. The specific method used for drug delivery and the dosage of immunosuppressants administered throughout disease treatment were individualized according to the patient's condition. The survival status was determined by telephone interview or through evaluation of the medical record. All patients were followed up at 1 year or until they met the end-point of death up to January 2019. These patients were included in for survival analysis. To calculate the survival rate, an end-point of either the date of death or the last date of follow-up at the outpatient clinic was recorded.

Statistical analysis. Statistical analysis was performed with SPSS version 24.0 (IBM Corp.). Continuous variables are expressed as the mean \pm standard deviation. Normally distributed data were compared using Student's t-test or one-way ANOVA followed by the least-significant difference post-hoc test. Non-normally distributed data are suitably expressed as the median (range or 25/75th percentile) and compared using Mann-Whitney U tests. Categorical variables are expressed as $\mathrm{n}(\%)$ and compared using the $\chi^{2}$ test. Survival rates were calculated with the Kaplan-Meier method (log rank test). The primary end-point was all-cause death. Rates of survival at 1,3 and 5 years were also determined. Univariate and multivariate logistic regression analysis with the Cox proportional hazards model was used to identify independent factors influencing mortality. The results are presented as hazard ratios (HRs) with $95 \%$ confidence intervals (CIs). $\mathrm{P}<0.05$ was considered to indicate statistical significance. The figure was prepared with GraphPad Prism 8 (GraphPad Software, Inc.).

\section{Results}

Comparison of demographic and baseline clinical characteristics among groups. The demographic and baseline clinical characteristics were compared among patients with SLE-PH, SSc-PH and pSS-PH. Patients with SSc were most likely to have PH [64/497 (12.9\%)], followed by those with pSS [34/523 (6.5\%)] and then by those with SLE [120/3,133 (3.8\%)]. Among the 218 patients with CTD-PH included in the present study, SLE-PH was most common (54.8\%), followed by SSc (29.2\%) and pSS (16.0\%). Demographic data, baseline clinical characteristics and treatments are presented in Table I. The mean age at the time of $\mathrm{PH}$ diagnosis was $47.47 \pm 38.70$ years. Patients with SLE-PH were younger $(40.54 \pm 49.82$ years) than those with SSc-PH $(58.02 \pm 10.93$ years; $\mathrm{P}<0.05)$ and patients with SSc-PH were younger than those with pSS-PH $(52.09 \pm 16.08$ years; $P>0.05)$. The overall study group included more females than males ( 83.9 vs. $16.1 \%$ ), with a significantly different sex ratio. There were more females in the SLE-PH than SSc-PH the group (92.5 vs. $68.8 \%$; $\mathrm{P}<0.05)$. The overall median duration of CTDs was 12 mo (range, 1-336 mo) and the median duration was by far the highest in patients with SSc-PH. The median duration of symptoms prior to the diagnosis of PH was 3 mo (range, 1-168 mo) and the median duration was highest in patients with pSS-PH. The rate of smoking was highest in patients with SSc-PH among all study groups. A total of 189 patients with CTD (86.7\%) had PH-related symptoms. The most common clinical symptom of CTD-PH was dyspnea (65.6\%), followed by cough (51.4\%), expectoration (39.9\%), edema (34.4\%), chest tightness (25.7\%) and chest pain (14.2\%). Raynaud's phenomenon appeared in $38.5 \%$ of patients with CTD-PH and was the most common symptom in patients with SSc-PH. Among all patients with CTD-PH, $42.2 \%$ were classified as WHO-FC III/IV (53.2\% of patients with SSc-PH, $44.2 \%$ of those with SLE-PH and 35.3\% of those with pSS-PH). In addition, patients with SSc-PH had the highest prevalence of lung involvement (81.2\%), followed by patients with pSS (58.8\%) and then by patients with SLE (30.0\%). When biochemical parameters were compared among the CTD-PH groups (data without significance were not shown), alkaline phosphatase (ALP) levels exhibited a significant difference. Although ALP levels were higher in the pSS-PH group than those in the SLE-PH and SSc-PH groups $(\mathrm{P}<0.05)$, the mean values remained within normal limits. Levels of NT-proBNP were elevated to various degrees in all three groups, with the greatest increase observed in the SLE-PH group. The level of IgG was higher in the pSS-PH group than that in the other two groups $(\mathrm{P}<0.05)$, likely due to the hyperglobulinemia associated with pSS. The antinuclear antibody $(\mathrm{Ab})$ and anti-extractable nuclear antigen $\mathrm{Ab}$ staining patterns were consistent with the characteristics of CTD. Pulmonary arterial pressure was mildly to moderately elevated in $80.3 \%$ of patients with CTD-PH. PASP was higher in the pSS group than in the SLE or SSc groups $(\mathrm{P}<0.05)$. Pericardial effusion was more common in patients with SLE-PH due to the nature of the disease. However, no differences among the 
Table I. Comparison of demographic and baseline clinical characteristics among patients with different types of CTD-PH.

\begin{tabular}{|c|c|c|c|c|c|}
\hline Item & Total $(n=218)$ & SLE-PH (n=120) & SSc-PH (n=64) & pSS-PH (n=34) & P-value \\
\hline Prevalence of $\mathrm{PH}^{\mathrm{a}}$ & $218 / 4,153(5.2)$ & $120 / 3,133(3.8)$ & 64/497 (12.9) & $34 / 523(6.5)$ & \\
\hline Age (years) & $47.47 \pm 38.70$ & $40.54 \pm 49.82$ & $58.02 \pm 10.93^{\mathrm{b}}$ & $52.09 \pm 16.08$ & 0.001 \\
\hline Female & $183(83.9)$ & $111(92.5)$ & $44(68.8)$ & $28(82.4)$ & $<0.001$ \\
\hline Duration of CTDs (mo) & $12(1-336)$ & $9.5(1-336)$ & $21.5(1-240)^{\mathrm{b}}$ & $12(1-108)$ & 0.007 \\
\hline $\begin{array}{l}\text { Duration from symptom } \\
\text { onset to } \mathrm{PH} \text { diagnosis (mo) }\end{array}$ & $3(1-168)$ & $2(1-84)$ & $7(1-108)$ & $12(1-168)$ & $<0.001$ \\
\hline Smoking & $20(9.2)$ & $3(1.7)$ & $15(23.4)^{\mathrm{b}}$ & $2(5.9)^{\mathrm{c}}$ & $<0.001$ \\
\hline $\operatorname{BMI}\left(\mathrm{kg} / \mathrm{m}^{2}\right)$ & $20.63 \pm 2.97$ & $20.65 \pm 3.04$ & $20.50 \pm 2.93$ & $20.79 \pm 2.84$ & 0.977 \\
\hline Hypertension & $45(20.6)$ & $21(17.5)$ & $18(28.1)$ & $6(17.6)$ & 0.213 \\
\hline Dyspnea & 143 (65.6) & $71(49.7)$ & $46(71.9)$ & $26(65.6)$ & 0.078 \\
\hline Cough & $112(51.4)$ & $63(52.5)$ & $34(53.1)$ & $15(44.1)$ & 0.652 \\
\hline Expectoration & 87 (39.9) & $44(36.7)$ & $31(48.4)$ & $12(36.7)$ & 0.250 \\
\hline Raynaud's phenomenon & $84(38.5)$ & $25(20.8)$ & $52(81.2)^{\mathrm{b}}$ & $7(20.6)^{\mathrm{c}}$ & $<0.001$ \\
\hline Edema & $75(34.4)$ & $56(46.7)$ & $16(25.0)^{\mathrm{b}}$ & $3(8.8)^{\mathrm{b}}$ & $<0.001$ \\
\hline Chest tightness & $56(25.7)$ & $35(29.2)$ & $13(20.3)$ & $8(23.5)$ & 0.404 \\
\hline Chest pain & $31(14.2)$ & $20(16.7)$ & $5(7.8)$ & $6(17.6)$ & 0.215 \\
\hline Syncope & $6(2.8)$ & $5(42.0)$ & $0(0.0)$ & $1(2.9)$ & 0.259 \\
\hline WHO-FC & & & & & 0.297 \\
\hline I & $79(36.2)$ & $48(40.0)$ & $23(35.9)$ & $8(23.5)$ & \\
\hline II & 47 (21.6) & $19(15.8)$ & $14(21.9)$ & $14(41.2)$ & \\
\hline III & $53(24.3)$ & $26(21.7)$ & $16(25.0)$ & $11(32.4)$ & \\
\hline IV & $39(17.9)$ & $27(22.5)$ & $1(28.2)$ & $1(2.9)$ & \\
\hline Lung involvement & $108(49.5)$ & $36(30.0)$ & $52(81.2)^{\mathrm{b}}$ & $20(58.8)^{\mathrm{b}, \mathrm{c}}$ & $<0.001$ \\
\hline $\operatorname{ALP}(\mathrm{U} / \mathrm{l})$ & $73.82 \pm 58.21$ & $66.94 \pm 39.93$ & $75.62 \pm 37.38$ & $95.81 \pm 116.13^{\mathrm{b}, \mathrm{c}}$ & 0.046 \\
\hline NT-proBNP (pg/ml) & $\begin{array}{c}2,761.5 \\
(966.3,10,816.0)\end{array}$ & $\begin{array}{c}4,411.5 \\
(2,303.5,14,065.0)\end{array}$ & $\begin{array}{c}1,333.0 \\
(181.2,10,342.0)^{\mathrm{b}}\end{array}$ & $\begin{array}{c}811.0 \\
(472.8,2,682.0)^{\mathrm{a}}\end{array}$ & 0.004 \\
\hline $\operatorname{IgG}(\mathrm{g} / \mathrm{l})$ & $\begin{array}{c}15.18 \\
(11.64,21.06)\end{array}$ & $\begin{array}{c}15.02 \\
(11.33,21.61)\end{array}$ & $\begin{array}{c}14.75 \\
(11.55,17.61)\end{array}$ & $\begin{array}{c}18.13 \\
(13.83,28.69)^{b, c}\end{array}$ & 0.065 \\
\hline \multicolumn{6}{|l|}{ Auto-Abs } \\
\hline ANA & $211(96.8)$ & $117(97.5)$ & $61(91.3)$ & $33(97.1)$ & 0.722 \\
\hline Anti-centromere $\mathrm{Ab}$ & $13(6.0)$ & $1(0.8)$ & $9(14.1)^{\mathrm{b}}$ & $3(8.8)^{\mathrm{b}, \mathrm{c}}$ & 0.001 \\
\hline Anti-nRNP/Sm Ab & $81(37.2)$ & $65(54.2)$ & $5(7.8)^{b}$ & $11(32.4)^{\mathrm{b}, \mathrm{c}}$ & $<0.001$ \\
\hline Anti-phospholipid Ab & $5(2.3)$ & $4(3.3)$ & $0(0)$ & $1(3.3)$ & 0.335 \\
\hline Glucocorticoids & $211(96.8)$ & $118(98.3)$ & $61(95.3)$ & $32(94.1)$ & 0.343 \\
\hline Immunosuppressants & $201(92.2)$ & $112(93.3)$ & $59(92.2)$ & $30(88.2)$ & 0.646 \\
\hline \multicolumn{6}{|l|}{ Vasodilator therapy } \\
\hline Use of medications & $94(43.1)$ & $48(40.0)$ & $32(50)$ & $14(41.2)$ & 0.414 \\
\hline Combination therapy & 19 (20.4) & $11(23.4)$ & $3(9.4)$ & $5(35.7)$ & 0.097 \\
\hline
\end{tabular}

${ }^{\mathrm{a} A m o n g}$ patients with that type of $\mathrm{CTD}$; ${ }^{\mathrm{b}} \mathrm{P}<0.05 \mathrm{vs}$. SLE-PH; ${ }^{\mathrm{P}} \mathrm{P}<0.05 \mathrm{vs}$. SSc-PH. Values are expressed as the mean \pm standard deviation, $\mathrm{n}(\%)$ or the median (range/the 25 and 75th percentiles). Ab, antibody; ALP, alkaline phosphatase (normal range, 35-100 U/l); ANA, antinuclear Ab; BMI, body mass index; CTD, connective tissue disease; IgG, immunoglobulin G (normal range, 8-18 g/l); mo, months; NT-proBNP, N-terminal pro-brain natriuretic peptide (normal range, $0-125 \mathrm{pg} / \mathrm{ml}$ ); $\mathrm{PH}$, pulmonary hypertension; pSS, primary Sjögren's syndrome; SLE, systemic lupus erythematosus; SSc, systemic sclerosis; WHO-FC, World Health Organization-function class; nRNP/Sm, nuclear ribonucleoprotein/Smith.

groups were observed in the degree of PH divided by the PASP or parameters of right-heart morphology (right atrial and ventricular enlargement, main pulmonary artery widening). The rate of left ventricular compliance decrease was significantly different among the groups (especially in the SSc-PH group; $\mathrm{P}<0.05$ ) (Table II).
Comparison of demographic and baseline clinical characteristics between survivors and non-survivors. Symptoms of dyspnea, expectoration and chest tightness were more common among non-survivors than survivors. The duration of symptoms prior to diagnosis of $\mathrm{PH}$ was longer in non-survivors (median, 8 mo; range, 1-168 mo) than in survivors (median, 2 mo; range, 1-144 mo). The 
Table II. Comparison of baseline echocardiographic characteristics among groups of patients with connective tissue disease-PH.

\begin{tabular}{|c|c|c|c|c|c|}
\hline Item & Total $(n=218)$ & SLE-PH (n=120) & SSc-PH $(n=64)$ & pSS-PH (n=34) & P-value \\
\hline PASP (mmHg) & $56.28 \pm 21.54$ & $56.54 \pm 21.29$ & $50.16 \pm 14.39^{\mathrm{a}}$ & $66.91 \pm 28.82^{\mathrm{a}, \mathrm{b}}$ & 0.001 \\
\hline Degree of $\mathrm{PH}$ & & & & & 0.146 \\
\hline Mild & $114(52.3)$ & $62(51.7)$ & $39(60.9)$ & $13(38.2)$ & \\
\hline Moderate & $61(28.0)$ & $31(25.8)$ & $18(28.1)$ & $12(35.3)$ & \\
\hline Severe & $43(19.7)$ & $27(22.5)$ & $7(10.9)$ & $9(26.5)$ & \\
\hline Pericardial effusion & $134(61.5)$ & $93(77.5)$ & $29(45.3)^{\mathrm{a}}$ & $12(35.3)^{\mathrm{a}}$ & $<0.001$ \\
\hline Right atrium enlargement & $58(27.1)$ & $36(30.0)$ & $12(18.8)$ & $11(32.4)$ & 0.197 \\
\hline Right ventricle enlargement & $52(23.9)$ & $30(25.0)$ & $11(17.2)$ & $11(32.4)$ & 0.223 \\
\hline Main pulmonary artery widening & $73(33.5)$ & $47(39.2)$ & $22(34.4)$ & $13(38.2)$ & 0.813 \\
\hline Decreased left ventricular compliance & $103(47.2)$ & $46(55.5)$ & $44(68.8)^{\mathrm{a}}$ & $14(38.2)^{\mathrm{b}}$ & $<0.001$ \\
\hline $\mathrm{EF}(\%)$ & $67.7 \pm 10.47$ & $66.65 \pm 9.81$ & $69.56 \pm 11.74$ & $66.40 \pm 5.31$ & 0.251 \\
\hline
\end{tabular}

${ }^{\mathrm{a}} \mathrm{P}<0.05$ vs. SLE-PH; ${ }^{\mathrm{b}} \mathrm{P}<0.05$ vs. SSc-PH. Values are expressed as the mean \pm standard deviation or $\mathrm{n}(\%)$. EF, ejection fraction; PASP, pulmonary arterial systolic pressure; $\mathrm{PH}$, pulmonary hypertension; pSS, primary Sjögren's syndrome; SLE, systemic lupus erythematosus; SSc, systemic sclerosis.

proportion of patients with WHO-FC III/IV in the non-survivor group $(58.9 \%)$ was significantly higher than the proportion in the survivor group (35.1\%; $\mathrm{P}=0.003)$. Compared with survivors, non-survivors had higher levels of NT-proBNP (Though with was not significant) and ALP, likely due to right ventricular dysfunction being more common among non-survivors than among survivors $(6,7)$. Lung involvement was also more common among non-survivors compared with survivors (44.4 vs. 58.9\%, $\mathrm{P}=0.075$; Table III). In the subgroup analysis of SSc-PH and pSS-PH, ILD HRCT scores were higher among non-survivors than among survivors (SSc, $20.33 \pm 3.92$ vs. $12.72 \pm 8.95$; pSS, $31.43 \pm 14.95$ vs $13.5 \pm 10.24$; $\mathrm{P}<0.05$; data not shown). Compared with CTD-PH survivors, non-survivors had higher PASP $(\mathrm{P}=0.001)$ and a higher proportion of cases with moderately to severely elevated pulmonary arterial pressure $(\mathrm{P}=0.001)$. Non-survivors also had a higher prevalence of right atrial enlargement and right ventricular enlargement $(\mathrm{P}<0.05$; Table IV).

Analysis of the impact of CTD disease activity on prognosis for patients with PH indicated that the SLEDAI scores were suggestive of high activity in both survivors and non-survivors, with no significant difference between groups (15.32 \pm 6.66 vs. $16.40 \pm 1.48 ; \mathrm{P}=0.528$; data not shown). Among the 64 patients with SSc-PH included in the study, $22(34.3 \%)$ had lcSSc and $42(65.6 \%)$ had dcSSc. Among the 47 patients with SSc-PH who were followed up, there was no significant difference in mortality between the lcSSc and dcSSc cases $[43.8 \%(7 / 16)$ vs. $45.2 \%(14 / 31)$, respectively; $\mathrm{P}=0.927]$, nor was there any significant difference between survivors and non-survivors in terms of mRSS scores for the degree of skin fibrosis $(26.88 \pm 10.45$ vs. $24.65 \pm 11.18$, respectively; $\mathrm{P}=0.493$ ) (above data not shown). Compared with non-survivors of pSS-PH, survivors of pSS-PH had higher levels of immunoglobulin $\mathrm{G}$ (IgG) [24.93 (15.16,32.43) vs. $13.33(11.69,15.11) \mathrm{g} / \mathrm{l}] ; \mathrm{P}=0.020]$ and ESSDAI [ $(22.88 \pm 10.90$ vs. $13.38 \pm 2.92 ; \mathrm{P}=0.025]$ (data not shown).

Determination of prognosis and predictors of mortality in patients with CTD-PH. Among the 218 patients included in the present study, $96.5 \%$ received glucocorticoids and $92.5 \%$ received immunosuppressive agents at the time of their diagnosis with CTD. A total of 72 patients (41.6\%) in 173 follow-up patients received specific pulmonary vasodilator therapy for $\mathrm{PH}$ and 14 patients $(8.1 \%)$ received combination therapy. No significant difference in the frequency with which these treatments were administered was observed between non-survivors and survivors (Table III). Short-term usage of targeted treatments was common and patients with CTD-PH were typically unable to continue targeted treatment for $>6$ mo.

The mean duration of follow-up after $\mathrm{PH}$ diagnosis was $26.17 \pm 20.66$ months. A total of 173 patients were followed up, including 98 patients with SLE-PH, 47 with SSc-PH and 28 with $\mathrm{pSS}-\mathrm{PH}$. The rates of mortality during the follow-up period were $26.3,48.8$ and $41.7 \%$, respectively. The overall survival rates at 1,3 and 5 year were $81.4,72.4$ and $56.9 \%$, respectively. The rate of survival tended to be lowest among patients with SSc (75.3, 63.0, 50.1\%), followed by patients with pSS (73.7, 59.7 and $59.7 \%$ ) and then by patients with SLE (84.7, 77.9 and 58.9\%); however, there was no significant difference among these three subgroups of patients with CTD-PH ( $\mathrm{P}=0.177$; Fig. 1).

Univariate Cox regression analysis of risk factors for mortality among patients with CTD-PH identified age $\geq 50$ years, $\mathrm{PH}$ severity, enlargement of the right ventricle, WHO-FCIII/IV, dyspnea and expectoration as influencing variables. However, in the multivariate analysis, only age $\geq 50$ years was an independent risk factor for mortality (Table V).

\section{Discussion}

In the present study, the clinical manifestations and survival rates associated with three major CTDs accompanied by $\mathrm{PH}$ were investigated. The study included patients from southwestern China who had been diagnosed with the condition based on the results of echocardiography, with the aim to screen and detect early-stage $\mathrm{PH}$. The prevalence of $\mathrm{PH}$ was highest among patients with SSc, while the SLE group accounted for the largest population among patients with 
Table III. Comparison of demographic and baseline clinical characteristics in CTD-PH survivors and non-survivors.

\begin{tabular}{|c|c|c|c|c|}
\hline Item & Total $(n=173)$ & Survivors $(\mathrm{n}=117)$ & Non-survivors $(\mathrm{n}=56)$ & P-value \\
\hline Age (years) & $47.47 \pm 38.70$ & $48.04 \pm 50.07$ & $50.46 \pm 18.61$ & 0.727 \\
\hline Female sex & $146(84.4)$ & $100(85.5)$ & $46(82.1)$ & 0.573 \\
\hline Duration of CTDs (mo) & $12(1-336)$ & $12(1-336)$ & $14(1-132)$ & 0.215 \\
\hline $\begin{array}{l}\text { Duration from symptom onset } \\
\text { to } \mathrm{PH} \text { diagnosis (mo) }\end{array}$ & $3(1-168)$ & $2(1-144)$ & $8(1-168)$ & 0.024 \\
\hline Smoking & $16(9.2)$ & $7(6.0)$ & $9(16.1)$ & 0.032 \\
\hline $\operatorname{BMI}\left(\mathrm{kg} / \mathrm{m}^{2}\right)$ & $20.63 \pm 2.97$ & $20.75 \pm 2.80$ & $20.45 \pm 3.10$ & 0.523 \\
\hline Hypertension & $39(22.5)$ & $21(17.9)$ & $18(32.1)$ & 0.037 \\
\hline Dyspnea & $112(64.7)$ & $68(58.1)$ & $44(78.6)$ & 0.008 \\
\hline Cough & $87(50.3)$ & $54(46.2)$ & $33(58.9)$ & 0.116 \\
\hline Expectoration & 67 (38.7) & $38(32.5)$ & $29(51.8)$ & 0.015 \\
\hline Raynaud's phenomenon & $62(35.8)$ & $38(32.5)$ & $24(42.9)$ & 0.183 \\
\hline Edema & $54(31.2)$ & $34(29.1)$ & $20(35.7)$ & 0.377 \\
\hline Chest tightness & $44(25.4)$ & $24(20.5)$ & $20(35.7)$ & 0.032 \\
\hline Chest pain & $25(14.5)$ & $18(15.4)$ & $7(12.5)$ & 0.614 \\
\hline Syncope & $5(2.9)$ & $2(1.7)$ & $3(5.4)$ & 0.330 \\
\hline WHO-FC & & & & 0.013 \\
\hline I & 65 (37.6) & $49(41.9)$ & $16(28.6)$ & \\
\hline II & $34(19.7)$ & $27(23.1)$ & $7(12.5)$ & \\
\hline III & $44(25.4)$ & $27(23.1)$ & $17(30.4)$ & \\
\hline IV & $30(17.3)$ & $14(12.0)$ & $16(28.6)$ & \\
\hline Lung involvement & $85(49.1)$ & $52(44.4)$ & $33(58.9)$ & 0.075 \\
\hline $\operatorname{ALP}(\mathrm{U} / \mathrm{l})$ & $73.82 \pm 58.21$ & $63.98 \pm 38.45$ & $86.32 \pm 91.73$ & 0.026 \\
\hline NT-proBNP (pg/ml) & $2,761.5(966.3,10,800.0)$ & $2,586.0(811.0,5,731.0)$ & $3,277.0(1,062.0,15,000)$ & 0.305 \\
\hline $\operatorname{IgG}(\mathrm{g} / \mathrm{l})$ & $15.18(11.64,21.06)$ & $3.37(1.62,3.37)$ & $15.01(10.67,20.05)$ & 0.419 \\
\hline \multicolumn{5}{|l|}{ Auto-Ab } \\
\hline ANA & $166(96.0)$ & $111(94.9)$ & $55(98.2)$ & 0.297 \\
\hline Anti-centromere $\mathrm{Ab}$ & $9(5.2)$ & $6(5.1)$ & $3(5.4)$ & 0.950 \\
\hline Anti-nRNP/Sm Ab & $67(38.4)$ & $48(41.0)$ & $19(33.9)$ & 0.370 \\
\hline Anti-phospholipid Ab & $5(2.9)$ & $4(3.4)$ & $1(1.8)$ & 1.000 \\
\hline Glucocorticoids & $167(96.5)$ & $115(98.3)$ & $52(92.9)$ & 0.087 \\
\hline Immunosuppressants & $160(92.5)$ & $111(94.9)$ & $49(87.5)$ & 0.121 \\
\hline \multicolumn{5}{|l|}{ Vasodilator therapy } \\
\hline Use of medications & 72 (41.6) & $49(41.9)$ & $23(41.1)$ & 0.920 \\
\hline Combination therapy & $14(8.1)$ & $9(7.8)$ & $5(8.9)$ & 0.793 \\
\hline
\end{tabular}

Values are expressed as the mean \pm standard deviation, $\mathrm{n}(\%)$ or the median (range/the 25 and 75 th percentiles). Ab, antibody; ALP, alkaline phosphatase; ANA, antinuclear Ab; BMI, body mass index; CTD, connective tissue disease; Ig, immunoglobulin; mo, months; NT-proBNP, N-terminal pro-brain natriuretic peptide; PH, pulmonary hypertension; WHO-FC, World Health Organization-function class; $\mathrm{nRNP} / \mathrm{Sm}$, nuclear ribonucleoprotein/Smith.

CTD-PH. Patients with CTD-PH who were screened early with Doppler echocardiography, particularly those with lung involvement, had less favorable prognoses. Therefore, early recognition by echocardiography and early treatment (particularly with vasodilators) should be attempted to counteract the poor prognosis exhibited by patients with CTD-PH.

The distribution of types of CTD associated with PAH varies across different regions. For instance, SSc-PAH predominates in Western cohorts (3). In Asian cohorts, SLE-PAH is the most common type of CTD associated with PAH and SSc-PAH is least common. One study of 129 Chinese patients with CTD-PAH reported that the type of CTD most commonly associated with PAH was SLE (49\%), followed by pSS (16\%), MCTD (9\%) and SSc (6\%) (6). A study performed at PUMCH suggested that patients with PAH were more likely to have SLE $(58.4 \%)$ than to have SSc $(26.3 \%)$ or pSS (15.3\%) (7). Similar results were reported in cohort studies based on data obtained from national registries in Japan (4) and South Korea (5). Consistent with the aforementioned results of Asian studies, the present study determined that the most common type of CTD-PH was SLE-PH (120/218, 54.8\%), while PH was most common among patients with SSc $(64 / 497,12.9 \%)$. 
Table IV. Comparison of baseline echocardiographic characteristics between connective tissue disease-PH survivors and non-survivors.

\begin{tabular}{lcccr}
\hline Item & Total $(\mathrm{n}=173)$ & Survivors $(\mathrm{n}=117)$ & Non-survivors $(\mathrm{n}=56)$ & P-value \\
\hline PASP (mmHg) & $56.28 \pm 21.54$ & $51.85 \pm 18.61$ & $63.46 \pm 23.02$ & 0.001 \\
Degree of PH & & & & 0.009 \\
Mild & $95(55.2)$ & $74(63.2)$ & $21(37.5)$ & \\
Moderate & $46(26.7)$ & $26(22.2)$ & $20(35.7)$ & \\
Severe & $31(18.0)$ & $17(14.6)$ & $15(26.8)$ & 0.438 \\
Pericardial effusion & $103(59.5)$ & $72(61.5)$ & $31(55.4)$ & 0.027 \\
Right atrium enlargement & $49(28.3)$ & $27(23.1)$ & $22(39.3)$ & 0.001 \\
Right ventricle enlargement & $44(25.4)$ & $21(17.9)$ & $23(41.1)$ & 0.378 \\
Main pulmonary artery widening & $66(38.2)$ & $42(35.9)$ & $32(57.1)$ & 0.060 \\
Decreased left ventricular compliance & $81(46.8)$ & $49(41.9)$ & $67.93 \pm 12.55$ & 0.511 \\
EF $(\%)$ & $67.70 \pm 10.48$ & $66.49 \pm 10.33$ & \\
\hline
\end{tabular}

Values are expressed as the mean \pm standard deviation or $\mathrm{n}(\%)$. EF, ejection fraction; PASP, pulmonary arterial systolic pressure; PH, pulmonary hypertension.

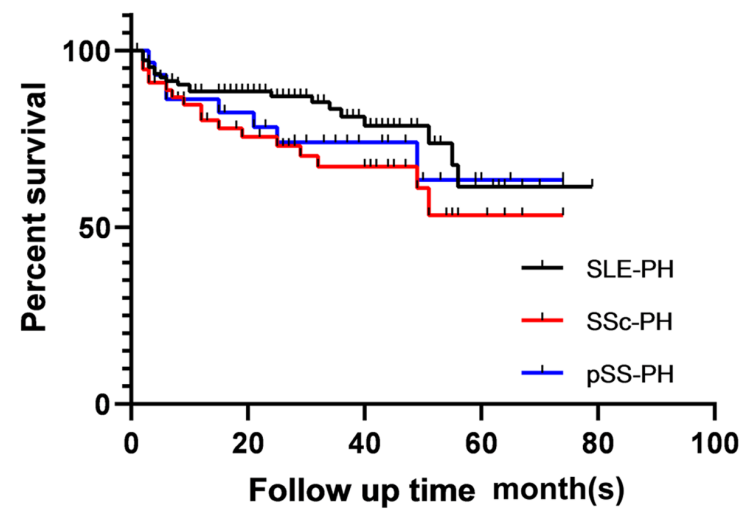

Figure 1. Kaplan-Meier survival analysis of patients with three major types of connective tissue disease-PH. PH, pulmonary hypertension; pSS, primary Sjögren's syndrome; SLE, systemic lupus erythematosus; SSc, systemic sclerosis.

In terms of survival, the present results indicated that disparities in disease distribution did not affect the risk of death. The 1-, 3- and 5-year survival rates among patients with CTD-PH were $81.4,72.4$ and $56.9 \%$, respectively, in accordance with the results previously reported by Hao et al and Zhao et al $(6,7)$. However, the survival rates reported in the present study are higher than those reported in the REVEAL study (21) and in the study by Condliffe et al (9). These differences in survival rates may reflect variations in the prevalence of CTDs.

Prognosis appeared to be most favorable among patients with SLE-PH and poorer among patients with SSc-PH as compared with patients with PH with other types of CTDs. Several reasons may account for this difference. In the present study, mean patient age and CTD disease duration were lower among patients with SLE-PH as compared with those in patients with SSc-PH. These patterns may reflect differences in the pathogenesis of inflammation and immune system dysfunction or differences in the response to immunosuppression therapies $(7,22)$. The pathological features of SLE are inflammatory lesions in small vessels throughout the circulatory system. Pulmonary hypertension in patients with SLE may be caused by acute inflammatory cell infiltration, vasospasm or microthrombus formation. Therefore, pulmonary arterial pressure may decrease or return to normal after active immunosuppression and anticoagulation in patients with SLE-PH. Patients with SSc-PH exhibit vascular lesion reactions, including chronic inflammatory cell infiltration, vascular endothelial damage, vascular remodeling and angiostenosis, all of which result in irreversible pathologic changes in pulmonary vessels. These patients may also respond poorly to immunosuppressive treatment (22). Finally, lung involvement has varying effects on prognosis in patients with CTD-PH $(6,7)$. Lung involvement that occurs in SLE is more likely to appear as acute interstitial pneumonia, diffuse alveolar hemorrhage with lupus pneumonia or acute pulmonary embolism caused by antiphospholipid syndrome. These conditions may be relieved or reversed by immunosuppressive treatment (22). However, chronic pulmonary fibrosis is the chronic lung lesion type that most commonly leads to pSS-PH and SSc-PH. This condition is also less responsive to immunosuppressive therapy $(6,7,22)$.

The present study indicated that pulmonary arterial pressure was typically mildly to moderately elevated in patients with CTD-PH. In addition, PASP was higher in the pSS group than in the SLE and SSc groups. Higher levels of IgG and ESSDAI were observed in pSS-PH survivors; CTD disease activity (SLEDAI in SLE, mRSS in SSc and ESSDAI in pSS) decreased after the administration of immunosuppressive therapy. It is noteworthy that PASP was higher in the pSS group than in the SLE and SSc groups, which was similar to the result in the study by Zhao et al (7), in which patients with pSS-PAH had worse hemodynamic profiles on diagnostic RHC than the SLE-PAH and SSc-PAH groups, with significantly higher mean right atrial pressure, mean pulmonary artery pressure and pulmonary vascular disease values and a lower cardiac index. The underlying mechanism is likely related to immune pathogenesis. An immunofluorescence study by Zhao et al (7) 
Table V. Univariate and multivariate analysis of predictors of mortality in patients with CTD-PH.

\begin{tabular}{|c|c|c|c|c|}
\hline \multirow[b]{2}{*}{ Factor } & \multicolumn{2}{|c|}{ Univariate analysis } & \multicolumn{2}{|c|}{ Multivariate analysis } \\
\hline & $\mathrm{HR}(95 \% \mathrm{CI})$ & P-value & $\operatorname{HR}(95 \% \mathrm{CI})$ & P-value \\
\hline Female sex & $0.79(0.40-1.56)$ & 0.496 & & \\
\hline Age $\geq 50$ years & $1.721(1.018-2.911)$ & 0.043 & $1.766(1.107-3.065)$ & 0.043 \\
\hline Duration of CTDs $\geq 5$ years & $0.630(0.308-1.286)$ & 0.204 & & \\
\hline Duration of symptom onset to $\mathrm{PH}$ diagnosis & $1.003(0.994-1.013)$ & 0.501 & & \\
\hline \multicolumn{5}{|l|}{ Type of CTDs } \\
\hline SLE, SSc and pSS & $1.251(0.899-1.74)$ & 0.184 & & \\
\hline SLE vs. non-SLE & $1.594(0.914-2.700)$ & 0.083 & & \\
\hline SSc vs. non-SSc & $1.568(0.913-2.695)$ & 0.103 & & \\
\hline Degree of PH (high, moderate and mild) & $1.588(1.160-2.172)$ & 0.004 & $1.333(0.086-2.065)$ & 0.199 \\
\hline Raynaud's phenomenon & $0.764(0.450-1.298)$ & 0.320 & & \\
\hline Dyspnea & $2.037(1.075-3.860)$ & 0.029 & $1.131(0.506-2.577)$ & 0.764 \\
\hline Expectoration & $1.704(1.008-2.881)$ & 0.047 & $1.372(0.797-2.363)$ & 0.254 \\
\hline Pericardial effusion & $0.799(0.471-1.354)$ & 0.799 & & \\
\hline Right atrium enlargement & $1.683(0.984-2.88)$ & 0.057 & & \\
\hline Right ventricle enlargement & $2.164(1.269-3.689)$ & 0.005 & $1.433(0.684-2.999)$ & 0.340 \\
\hline Main pulmonary artery widening & $1.2(0.692-2.081)$ & 0.515 & & \\
\hline Lung involvement & $1.456(0.854-2.484)$ & 0.168 & & \\
\hline SSc and pSS-ILD & $1.167(0.502-2.711)$ & 0.720 & & \\
\hline WHO-FC III/IV vs. I/II & $2.213(1.298-3.773)$ & 0.004 & $1.414(0.715-2.798)$ & 0.320 \\
\hline Use of vasodilator therapy & $0.943(0.553-1.608)$ & 0.829 & & \\
\hline Combination vasodilator therapy & $1.153(0.460-2.891)$ & 0.762 & & \\
\hline
\end{tabular}

CTD, connective tissue disease; HR, hazard ratio; Ig, immunoglobulin; ILD, interstitial lung disease; NT-proBNP, N-terminal pro-brain natriuretic peptide; PH, pulmonary hypertension; pSS, primary Sjögren's syndrome; SLE, systemic lupus erythematosus; SSc, systemic sclerosis; WHO-FC, World Health Organization-function class.

demonstrated the deposition of immune system components in the muscular-type arteries of patients with pSS-PAH, indicating the presence of an immune complex-mediated injury. This former study postulated the hypothesis that endothelial damage, immune complex accumulation, necrotic vasculitis and imbalances in the levels of endothelium-derived vasoactive molecules contribute to the pathogenesis of pSS-PAH. The pathogenesis of pSS-PAH is similar to that of the acute inflammatory lesions present in patients with SLE-PAH, which may respond well to treatment (7). As with the results of previous studies $(6,7)$, patients with SLE were younger than those with pSS and SSc in the current study, and age was an important contributor to mortality, as older patients had a higher mortality rate. Delays in diagnosis may also account for differences in survival rates between patients with SLE-PH and pSS-PH. Additional studies will be necessary to better understand existing barriers to the early diagnosis of $\mathrm{PH}$ in patients with pSS (7).

Regarding predictors of mortality, previous studies suggested that poor heart function indices, poor hemodynamics, lower exercise capacity, lower mixed venous oxygen saturation, WHO-FC III/IV and elevated NT-proBNP were all significant risk factors among patients with CTD-PAH (3-5). The present results indicated that age $\geq 50$ years, severity of $\mathrm{PH}$, enlargement of the right ventricle, WHO-FC III/IV, dyspnea and expectoration were associated with poor survival. Age $\geq 50$ years was the only independent risk factor for mortality. There is currently no consensus regarding prognostic risk factors such as age, Raynaud's phenomenon, pericardial effusion and ILD.

Age is well recognized as a significant predictor of survival in patients with CTD-PH. The UK's national registry of all incident cases of CTD-PAH indicated that patients $<60$ years of age had better survival rates than patients aged $\geq 70$ years (9). A study based on data from the French SSc-PAH registry that was published in 2012 suggested that the risk of death of patients increased by 1.05 -fold for each additional year of age at the time of PAH diagnosis (23). Analysis of the patient data in South Korea's nationwide registry of patients with CTD-PH (as detected by echocardiography) suggested that age $>60$ years was a risk factor for mortality (5). In the present study, multivariate analysis revealed that age $\geq 50$ years was the only independent risk factor for mortality. These discrepancies among studies may reflect differences in sample size, CTD distribution and diversity in populations. Hence, further large-cohort studies that distinguish between CTD-PAH patient subgroups are required to verify the effect of age on survival of patients with CTD-PAH.

Raynaud's phenomenon, fingertip ulcer/pit and gangrene are examples of direct evidence of vascular disease. Raynaud's 
phenomenon is considered to be associated with CTD-PAH and is an independent risk factor for the onset of $\mathrm{PAH}$. Mechanistically, Raynaud's phenomenon is associated with systolic and diastolic dysfunction of the acral arteriole. When it affects the pulmonary artery, the condition is called 'pulmonary Raynaud's phenomenon' (24). The persistent vasospasm of pulmonary arterioles may result in pulmonary vascular remodeling and increased pulmonary vascular resistance (24). In the present study, Raynaud's phenomenon appeared to be more common in the group of CTD-PH non-survivors compared with survivors. However, this difference was not statistically significant, which was in accordance with the report by Zhao et al (7). Therefore, whether Raynaud's phenomenon is a prognostic risk factor for CTD-PH remains controversial.

Pleural effusion is considered to be a risk factor for PAH among patients with CTD. However, whether pleural effusion predicts survival remains controversial. A study by Hao et al (6) and one study based on data from the South Korean nationwide registry (5) suggested that patients with pleural effusion had poorer survival outcomes than patients without pleural effusion, highlighting that the condition may be a predictor of mortality among patients with CTD-PAH. However, one study of patients with SSc-PAH from France (23) and the study by Zhao et al (7) indicated no significant association between the prevalence of pleural effusion and survival of patients with CTD-PAH. The present study also failed to identify a significant association between pleural effusion and survival among patients with CTD-PAH. Additional studies will be required to further clarify these discrepant findings.

Lung involvement is common in patients with CTD. SLE tends to be complicated by ILD (usually non-specific interstitial pneumonia), diffuse hemorrhagic alveoli and acute lupus pneumonia, all of which respond well to immunosuppressive therapy. ILD (typically in the form of interstitial pneumonia) is more frequently encountered in patients with SSc or pSS, among whom immunosuppressive therapy is less effective. Mild PH is common among patients with severe interstitial lung disease. Hao et al (6) defined ILD-PH as moderate ILD based on the results of HRCT (1/3-2/3 of the lung field involved) in combination with a total lung capacity of $60 \%$ predicted via pulmonary function testing, or severe ILD based on the results of HRCT ( $>2 / 3$ of the lung field involved). The results of Hao et al (6) indicated that the prognosis of patients with CTD with ILD-PH was significantly less favorable than that of patients with isolated CTD-PAH in Chinese cohorts. Studies from the UK (9), US (21) and France (23) obtained similar results after comparing the survival of patients with ILD-PH or respiratory-associated $\mathrm{PH}$ with the survival of patients with isolated SSc-PAH. In the present study, lung involvement with CTD-PH appeared to be more common among non-survivors than among survivors, but the difference was not statistically significant. Further subgroup analysis of the combination of SSc-PAH and pSS-PAH suggested that the HRCT scores observed in non-survivors were higher than those in survivors. Hence, it may be concluded that severe ILD is associated with a poor prognosis.

Of note, the present study had certain limitations. First, $\mathrm{PH}$ was defined on the basis of PASP $>35 \mathrm{mmHg}$, as measured by
Doppler echocardiography but not by RHC. However, pulmonary arterial pressure (as measured by echocardiography) was indicated to be consistently associated with the hemodynamic index measured by RHC $(3,5,9,12-14)$. Echocardiography is a non-invasive diagnostic tool that provides a reasonably reliable and comprehensive assessment of the right heart and pulmonary circulation and may be used routinely for screening and diagnosing early PH. Furthermore, due to the lack of pulmonary function tests, it was not possible to compare ILD-PH and isolated PH. In addition, although $43.1 \%$ of patients included in the present study used pulmonary vasodilators and $20.4 \%$ received combination therapy, drug withdrawal was common. Beraprost was adopted as a specific pulmonary treatment in the present study; however, beraprost is less effective than other pulmonary vasodilators or combination therapy. No significant difference in the frequency with which these treatments were administered was observed between non-survivors and survivors. This result may reflect selection bias, as patients at higher risk of mortality were selected because they had higher rates of PASP and right ventricle dysfunction. It is also possible that short-term specific PAH treatment improved symptoms in patients with CTD only transiently without altering the overall prognosis. The poorer prognosis may reflect the fact that the patients in the present study who received pulmonary vasodilators were typically older patients with CTD-PH who had systemic disease, particularly myocardial involvement and comorbidities. However, even if a patient's condition worsened, there was no increase in the dose or administration of combined treatment due to the cost that would accrue to the patient and their family. Further investigation will be necessary to determine whether more aggressive management is able to improve the overall prognosis in such cases (23).

In conclusion, among these three major types of CTD-PH, SSc-PH had the highest prevalence. The overall prognosis for patients with CTD-PH remains poor. Patients with SLE-PH have the most favorable prognosis. Among the 3 types of CTD-PH, patients with SSc-PH have the worst outcomes. Age $\geq 50$ years is the only independent risk factor for mortality.

\section{Acknowledgements}

The authors would like to thank our radiology colleagues, Dr Kai Li and Dr Xuechun Guan (Department of Radiology, the First Affiliated Hospital of Guangxi Medical University), whose experience in interstitial lung diseases helped examine the HRCT results and assign HRCT scores.

\section{Funding}

This study was supported by grants from the National Natural Science Foundation (grant nos. 81860292 and 81760295) and Guangxi Medical and Health Technology Development and Promotion Project (grant no. S2018084).

\section{Availability of data and materials}

The datasets used and/or analyzed during the current study are available from the corresponding author on reasonable request. 


\section{Authors' contributions}

JP collected and analyzed the data, and wrote the manuscript. JW, FQ and FD collected the data, including demographic data, baseline clinical features, laboratory results and echocardiographic parameters. LL and CZ contributed to the conception, design and organization of the current study, and reviewed and revised the manuscript. LL and $\mathrm{CZ}$ confirm the authenticity of all the raw data. All authors read and approved the final version of the manuscript. All authors are fully responsible for all aspects of the study and the final manuscript. All authors agree to be accountable for all aspects of the work in ensuring that questions related to the accuracy or integrity of any part of the work are appropriately investigated and resolved.

\section{Ethics approval and consent to participate}

The study protocol was approved by the Ethics Committee of the First Affiliated Hospital of Guangxi Medical University (approval no. 2018-KY-National Natural Science Foundation-012). Written informed consent was obtained from all patients.

\section{Patient consent for publication}

Not applicable.

\section{Competing interests}

The authors declare that they have no competing interests.

\section{References}

1. Galiè N, Humbert M, Vachiery JL, Gibbs S, Lang I, Torbicki A, Simonneau G, Peacock A, Vonk Noordegraaf A, Beghetti M, et al: $2015 \mathrm{ESC} / \mathrm{ERS}$ guidelines for the diagnosis and treatment of pulmonary hypertension: The joint task force for the diagnosis and treatment of pulmonary hypertension of the European society of cardiology (ESC) and the European respiratory society (ERS) Endorsed by: Association for European paediatric and congenital cardiology (AEPC), international society for heart and lung transplantation (ISHLT). Eur Heart J 37: 67-119, 2016.

2. Simonneau G, Montani D, Celermajer DS, Denton CP, Gatzoulis MA, Krowka M, Williams PG and Souza R: Haemodynamic definitions and updated clinical classification of pulmonary hypertension. Eur Respir J 53: 1801913, 2019.

3. Thakkar V and Lau EM: Connective tissue disease-related pulmonary arterial hypertension. Best Pract Res Clin Rheumatol 30 22-38, 2016.

4. Shirai Y, Yasuoka H, Okano Y, Takeuchi T, Satoh T and Kuwana M: Clinical characteristics and survival of Japanese patients with connective tissue disease and pulmonary arterial hypertension: A single-centre cohort. Rheumatology (Oxford) 51: 1846-1854, 2012.

5. Kang KY, Jeon CH, Choi SJ, Yoon BY, Choi CB, Lee CH, Suh CH, Lee CW, Cho CS, Nam EJ, et al: Survival and prognostic factors in patients with connective tissue disease-associated pulmonary hypertension diagnosed by echocardiography: Results from a Korean nationwide registry. Int J Rheum Dis 20: 1227-1236, 2017.

6. Hao YJ, Jiang X, Zhou W, Wang Y, Gao L, Wang Y, Li GT, Hong T, Huo Y, Jing ZC and Zhang ZL: Connective tissue disease-associated pulmonary arterial hypertension in Chinese patients. Eur Respir J 44: 963-972, 2014.

7. Zhao J, Wang Q, Liu Y, Tian Z, Guo X, Wang H, Lai J, Huang C, Yang X, Li M and Zeng X: Clinical characteristics and survival of pulmonary arterial hypertension associated with three major connective tissue diseases: A cohort study in China. Int J Cardiol 236: 432-437, 2017.
8. Fei Y, Shi X, Gan F, Li X, Zhang W, Li M, Hou Y, Zhang X, Zhao Y, Zeng X and Zhang F: Death causes and pathogens analysis of systemic lupus erythematosus during the past 26 years. Clin Rheumatol 33: 57-63, 2014

9. Condliffe R, Kiely DG, Peacock AJ, Corris PA, Gibbs JS, Vrapi F, Das C, Elliot CA, Johnson M, DeSoyza J, et al: Connective tissue disease-associated pulmonary arterial hypertension in the modern treatment era. Am J Respir Crit Care Med 179: 151-157, 2009.

10. Fischer A, Denton CP, Matucci-Cerinic M, Gillies H, Blair C, Tislow $\mathrm{J}$ and Nathan SD: Ambrisentan response in connective tissue disease-associated pulmonary arterial hypertension (CTD-PAH)-A subgroup analysis of the ARIES-E clinical trial. Respir Med 117: 254-263, 2016.

11. Coghlan JG, Galiè N, Barberà JA, Frost AE, Ghofrani HA, Hoeper MM, Kuwana M, McLaughlin VV, Peacock AJ, Simonneau G, et al: Initial combination therapy with ambrisentan and tadalafil in connective tissue disease-associated pulmonary arterial hypertension (CTD-PAH): Subgroup analysis from the AMBITION trial. Ann Rheum Dis 76: 1219-1227, 2017.

12. Weatherald J, Montani D, Jevnikar M, Jaïs X, Savale L and Humbert M: Screening for pulmonary arterial hypertension in systemic sclerosis. Eur Respir Rev 28: 190023, 2019.

13. Shahane A: Pulmonary hypertension in rheumatic diseases: Epidemiology and pathogenesis. Rheumatol Int 33: 1655-1667, 2013.

14. Barst RJ, McGoon M, Torbicki A, Sitbon O, Krowka MJ, Olschewski H and Gaine S: Diagnosis and differential assessment of pulmonary arterial hypertension. J Am Coll Cardiol 43: (Suppl 12), 40S-47S, 2004.

15. Fortuna G and Brennan MT: Systemic lupus erythematosus: Epidemiology, pathophysiology, manifestations, and management. Dent Clin North Am 57: 631-655, 2013.

16. van den Hoogen F, Khanna D, Fransen J, Johnson SR, Baron M, Tyndall A, Matucci-Cerinic M, Naden RP, Medsger TA Jr, Carreira PE, et al: 2013 classification criteria for systemic sclerosis: An American College of rheumatology/European league against rheumatism collaborative initiative. Arthritis Rheum 65: 2737-2747, 2013.

17. Vitali C, Bombardieri S, Jonsson R, Moutsopoulos HM, Alexander EL, Carsons SE, Daniels TE, Fox PC, Fox RI, Kassan SS, et al: Classification criteria for Sjögren's syndrome: A revised version of the European criteria proposed by the American-European consensus group. Ann Rheum Dis 61: 554-558, 2002.

18. Goldin JG, Lynch DA, Strollo DC, Suh RD, Schraufnagel DE, Clements PJ, Elashoff RM, Furst DE, Vasunilashorn S, McNitt-Gray MF, et al: High-resolution CT scan findings in patients with symptomatic scleroderma-related interstitial lung disease. Chest 134: 358-367, 2008.

19. Tang J, Lei L, Pan J, Zhao C and Wen J: Higher levels of serum interleukin-35 are associated with the severity of pulmonary fibrosis and Th2 responses in patients with systemic sclerosis. Rheumatol Int 38: 1511-1519, 2018.

20. Seror R, Theander E, Brun JG, Ramos-Casals M, Valim V, Dörner T, Bootsma H, Tzioufas A, Solans-Laqué R, Mandl T, et al: Validation of EULAR primary Sjögren's syndrome disease activity (ESSDAI) and patient indexes (ESSPRI). Ann Rheum Dis 74: 859-866, 2015

21. Chung L, Liu J, Parsons L, Hassoun PM, McGoon M, Badesch DB, Miller DP, Nicolls MR and Zamanian RT: Characterization of connective tissue disease-associated pulmonary arterial hypertension from REVEAL: Identifying systemic sclerosis as a unique phenotype. Chest 138: 1383-1394, 2010.

22. Zanatta E, Polito P, Famoso G, Larosa M, De Zorzi E, Scarpieri E, Cozzi F and Doria A: Pulmonary arterial hypertension in connective tissue disorders: Pathophysiology and treatment. Exp Biol Med (Maywood) 244: 120-131, 2019.

23. Launay D, Sitbon O, Hachulla E, Mouthon L, Gressin V, Rottat L, Clerson P, Cordier JF, Simonneau G and Humbert M: Survival in systemic sclerosis-associated pulmonary arterial hypertension in the modern management era. Ann Rheum Dis 72: 1940-1946, 2013.

24. Callejas-Rubio JL, López-Pérez L, Moreno-Escobar E and Ortego-Centeno N: Raynaud's phenomenon and pulmonary arterial hypertension. Lupus 17: 355, 2008.

This work is licensed under a Creative Commons Attribution-NonCommercial-NoDerivatives 4.0 International (CC BY-NC-ND 4.0) License. 